\title{
A novel phosphatase cascade regulates differentiation in Trypanosoma brucei via a glycosomal signaling pathway
}

\author{
Balázs Szöör, ${ }^{1,4}$ Irene Ruberto, ${ }^{1}$ Richard Burchmore, ${ }^{2}$ and Keith R. Matthews ${ }^{1,3}$ \\ ${ }^{1}$ Centre for Immunity, Infection, and Evolution, Institute of Immunology and Infection Research, School of Biological Sciences, \\ University of Edinburgh, Edinburgh EH9 3JT; United Kingdom; ${ }^{2}$ Sir Henry Wellcome Proteomics Facility, University of Glasgow \\ G12 8QQ, United Kingdom
}

In the mammalian bloodstream, the sleeping sickness parasite Trypanosoma brucei is held poised for transmission by the activity of a tyrosine phosphatase, TbPTP1. This prevents differentiation of the transmissible "stumpy forms" until entry into the tsetse fly, whereupon TbPTP1 is inactivated and major changes in parasite physiology are initiated to allow colonization of the arthropod vector. Using a substrate-trapping approach, we identified the downstream step in this developmental signaling pathway as a DxDxT phosphatase, TbPIP39, which is activated upon tyrosine phosphorylation, and hence is negatively regulated by TbPTP1. In vitro, TbPIP39 promotes the activity of $T b P T P 1$, thereby reinforcing its own repression, this being alleviated by the trypanosome differentiation triggers citrate and cis-aconitate, generating a potentially bistable regulatory switch. Supporting a role in signal transduction, $T b P I P 39$ becomes rapidly tyrosine-phosphorylated during differentiation, and RNAimediated transcript ablation in stumpy forms inhibits parasite development. Interestingly, TbPIP39 localizes in glycosomes, peroxisome-like organelles that compartmentalize the trypanosome glycolytic reactions among other enzymatic activities. Our results invoke a phosphatase signaling cascade in which the developmental signal is trafficked to a unique metabolic organelle in the parasite: the glycosome. This is the first characterized environmental signaling pathway targeted directly to a peroxisome-like organelle in any eukaryotic cell.

[Keywords: Glycosome; peroxisome; signal transduction; phosphatase; Trypanosoma brucei; differentiation]

Supplemental material is available at http://www.genesdev.org.

Received November 26, 2009; revised version accepted April 22, 2010.

Developmental events in eukaryotic cells are often driven by a transmembrane signaling event, this being transduced via phosphorylation/dephosphorylation of signaling proteins to generate a cellular response. Such signaling events are particularly important in unicellular eukaryotes, which are required to react to external stimuli, either as part of an adaptive response to a changing environment, or through triggered developmental responses intrinsic to their normal life cycle progression. An excellent model for such developmentally regulated responses is the African trypanosome,Trypanosoma brucei (Fenn and Matthews 2007). These are important disease organisms of subSaharan Africa, where they generate significant problems for public health and economic welfare (Barrett et al. 2003). Furthermore, as highly diverged and evolutionarily ancient organisms (Sogin et al. 1986), they have provided

Corresponding authors.

${ }^{3}$ E-MAIL keith.matthews@ed.ac.uk; FAX 44-131-651-3670.

${ }^{4}$ E-MAIL Balazs.Szoor@ed.ac.uk; FAX 44-131-651-3670.

Article is online at http://www.genesdev.org/cgi/doi/10.1101/gad.570310. Freely available online through the Genes \& Development Open Access option. an important paradigm for organelle evolution and function in the eukaryotic cell (Hannaert et al. 2003; He et al. 2004, 2005; Broadhead et al. 2006; He 2007), exemplified by their use of extensive RNA editing for mitochondrial transcripts (Stuart et al. 2005), and by their possession of unusual peroxisome-like organelles, glycosomes (Opperdoes 1987). These compartmentalize the trypanosome glycolytic enzymes, which in other eukaryotes are cytosolic, thereby avoiding the lethal consequences of the "turbo design" of glycolysis (Teusink et al. 1998; Bakker et al. 2000). Glycosomes also contain enzymes required for ether lipid biosynthesis, the $\beta$ oxidation of fatty acids, as well as several additional activities (Michels et al. 2006; Opperdoes and Szikora 2006), although their precise enzymatic composition and number is regulated during the trypanosome life cycle (Michels et al. 2006).

The life cycle regulation of organellar function is a central component of the developmental biology of the trypanosome, which entails extensive cellular remodeling during passage from the mammalian blood to the midgut of the tsetse fly, the parasite's vector (Matthews 2005). These differentiation events are triggered by exposure 
of bloodstream parasites to citrate/cis-aconitate (CCA) (Czichos et al. 1986; Engstler and Boshart 2004). This signal is transduced via a family of surface transporters, PAD proteins (Dean et al. 2009), with signal recognition being promoted by temperature reduction to $20^{\circ} \mathrm{C}-$ conditions encountered upon uptake by feeding tsetse flies (Engstler and Boshart 2004). PAD proteins are expressed on the cell surface of stumpy forms, the stage of the bloodstream parasite population adapted for transmission to tsetse flies. Importantly, stumpy forms are held ready for this developmental change by the action of a tyrosine phosphatase, TbPTP1 (Szöör et al. 2006), until differentiation is triggered. Thus, the activity of TbPTP1 prevents stumpy forms from initiating differentiation and, when this enzyme is inactivated after exposure to either CCA or chemical inhibitors of PTPs, the cells differentiate from bloodstream to procyclic forms (Szöör et al. 2006). This places TbPTP1 at the head of an intracellular signaling pathway whose components are completely unknown.

Here we exploited a tyrosine phosphatase substrate selection strategy to identify the downstream step in the differentiation signaling pathway. This has identified a glycosomally targeted DxDxT family Ser/Thr phosphatase, invoking the presence of a phosphatase signaling cascade directing developmental regulation in trypanosomes. Our findings provide the first evidence of developmentally regulated signal transduction via the glycosome, and link the external differentiation stimulus through this signaling pathway to control of the trypanosome life cycle.

\section{Results}

TbPTP1 substrate is a novel DxDxT phosphatase, TbPIP39

To identify potential substrates of TbPTP1, we exploited the ability to generate mutant PTP enzymes, which bind but do not release substrates (Blanchetot et al. 2005). Specifically, His-tagged TbPTP1 was generated in which residue 199, the catalytic aspartic acid in the predicted WPD loop (Szöör et al. 2006), was mutated to alanine. This "substrate-trapping" mutant (TbPTP1-D199A) was bound to a His trap chelating column, and was used to select interacting proteins and substrates of TbPTP1 from stumpy cell lysates. Mass spectrometry reproducibly identified a selected protein encoded by a gene on chromosome 9, Tb09.160.4460, this being almost identical (339 out of 343 amino acids) to that encoded by its immediately downstream gene (Tb09.160.4480), invoking a gene duplication event. Tb09.160.4460 encodes a protein with a predicted molecular mass of $39 \mathrm{kDa}$, this being named TbPIP39 (TbPTP1-interacting protein, $39 \mathrm{kDa}$ ). The TbPIP39 gene is well conserved in those kinetoplastids that have been subject to genome sequence analysis, although in Trypanosoma cruzi, Trypanosoma vivax, and Leishmania major, it has not undergone gene duplication (Supplemental Fig. 1).

To confirm the interaction between TbPTP1 and TbPIP39, an antibody was raised to TbPIP39 and used to probe the eluate from stumpy cell lysates incubated with either His-tagged TbPTP1D199A or wild-type TbPTP1. Figure 1A demonstrates that the binding of TbPIP39 to the substrate-trapping TbPTP-D199A was much more effective than to the wild-type protein (Fig. 1A, lanes 2,3). To validate this interaction in vivo, transgenic bloodstream forms were generated ectopically expressing wildtype TbPTP1 or TbPTP1-D199A proteins, each with a C-terminal Ty1 epitope tag (Bastin et al. 1996). Since TbPIP39 expression is induced during differentiation (see later), cells were treated with cis-aconitate for $24 \mathrm{~h}$, and Tyl-specific antibody was used to immunoprecipitate TbPTP1. This revealed coimmunoprecipitation of TbPIP39 with the TbPTP-D199A substrate-trapping mutant, selection being blocked in the presence of a synthetic peptide comprising the Ty1 epitope (Fig. 1B, cf. lanes 3 and 5). Matching the in vitro pull-down assay, TbPIP39 was selected less efficiently with wild-type TbPTP1 (Fig. $1 \mathrm{~B}$, cf. lanes 5 and 10 ), supportive of the interaction being stabilized by the substrate-trapping mutation. Confirming
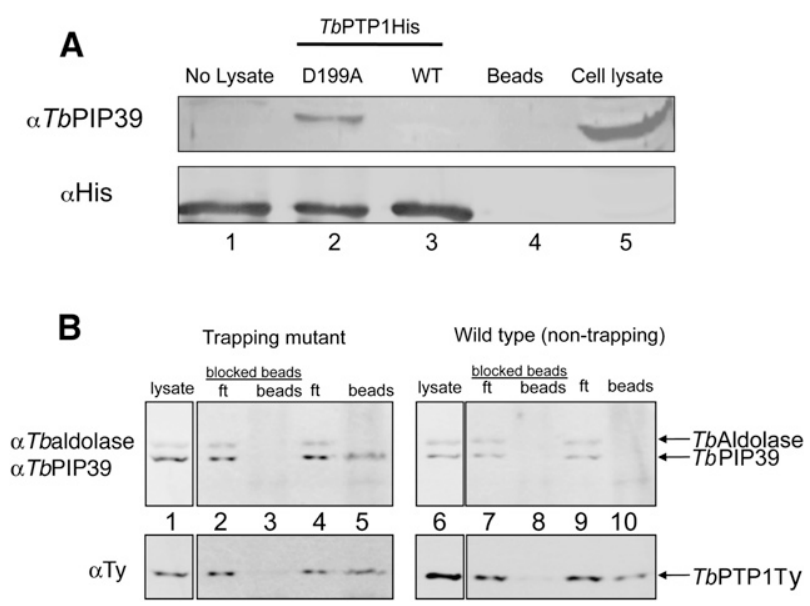

Figure 1. TbPTP1 interacts with TbPIP39 in vitro and in vivo. (A) Substrate-trapping selection of TbPIP39 by TbPTP1. Wildtype (WT) or substrate-trapping recombinant TbPTP1 (D199A) was expressed as a His-tagged fusion protein and then bound to a His trap chelating column. Lysate from $5 \times 10^{8}$ stumpy cells was then passed over the column in order to select interacting proteins. Bound material was eluted from the beads and reacted with either antibody specific for the His tag (detecting the TbPTP1 ligand), or TbPIP39. TbPIP39 was preferentially selected by the D199A TbPTP1 substrate-trapping mutant. (B) Wild-type or D199A TbPTP1-Ty was expressed in cultured bloodstream form T. brucei, which were then induced to differentiate to procyclic forms for $24 \mathrm{~h}$ by the addition of $6 \mathrm{mM}$ cisaconitate. Immunoprecipitation was then performed using the TY-1 epitope-specific antibody, which had been incubated either with ("blocked beads") or without a peptide sequence recognized by the antibody. Input cell lysate, flow-through $(\mathrm{ft})$, or precipitated material (beads) from each immunoprecipitation, either with or without blocking, was then reacted with the Ty1specific antibody (to detect the ectopically expressed TbPTP1 or D199A TbPTP1) or antibodies detecting TbPIP39 or aldolase (as a negative control). The D199A-trapping mutant of TbPTP1 preferentially selected TbPIP39, whereas aldolase was not selected. 
specificity, an unrelated protein, aldolase, was not coselected by immunoprecipitation of the substrate-trapping or wild-type TbPTP1 (Fig. 1B).

Examination of the sequence of TbPIP39 and its orthologous sequences in related kinetoplastids revealed the presence of a conserved predicted tyrosine phosphorylation site (Y278) toward the C terminus (Supplemental Fig. 1A). Although the trypanosome genome encodes no tyrosine-specific kinases (Parsons et al. 2005), a groupbased phosphorylation scoring analysis (Xue et al. 2005) predicted that the $\mathrm{Y} 278$ residue could be phosphorylated effectively in vitro by the human Gardner-Rasheed feline sarcoma viral (v-fgr) oncogene homolog FGR kinase. Confirming this, incubating recombinant TbPIP39 with FGR kinase rapidly generated tyrosine-phosphorylated TbPIP39 (Fig. 2A, left panel), whereas mutation of Y278 to phenylalanine (Y278F) in TbPIP39 prevented tyrosine phosphorylation under the same conditions (Fig. 2A, right panel). Tyrosine-phosphorylated TbPIP39 was then incubated with either wild-type TbPTP1, or a catalytically dead mutant of TbPTP1 in which the active site cysteine was mutated to serine (C229S). Figure 2B demonstrates that TbPIP39 was effectively dephosphorylated by wildtype TbPTP1, but not by the inactive C229S mutant, establishing that TbPIP39 not only interacted with substrate-trapping TbPTP1 in cell lysates, but that it could form a TbPTP1 substrate.

\section{Regulatory interactions between $\mathrm{TbPTP} 1$ and $\mathrm{Tb} P I P 39$}

The protein sequence of TbPIP39 suggested that it was a member of an unusual class of serine/threonine phosphatases, with a characteristic $\mathrm{DxDx}(\mathrm{T} / \mathrm{V})$ motif toward the $\mathrm{N}$ terminus of the predicted catalytic domain (Supplemental Fig. 1A). This family of proteins comprises FCP1/SCP1, responsible for the dephosphorylation of the eukaryotic C-terminal domain of RNA polymerase II (Kamenski et al. 2004), as well as several stress response phosphatases, typified by the yeast haloacid dehalogenasetype phosphatases PSR1/PSR2 (Siniossoglou et al. 2000) and a phosphatase required in nuclear membrane biogenesis, Dullard (Kim et al. 2007). The catalytic activity of this group of phosphatases depends on magnesium ions, requiring the integrity of the DxDxT motif as the intermediate phosphoryl acceptor (Collet et al. 1998). Consistent with this, the activity of recombinant TbPIP39 against the artificial phosphatase substrate pNPP was enhanced dramatically $(P<0.001)$ in the presence of $15 \mathrm{mM} \mathrm{Mg}^{2+}$ (Fig. 2C, columns 1,2). Moreover, when residues 55D and 57D were each mutated to glutamic acid (TbPIP39dEdE), no catalytic activity could be detected under the same conditions (Fig. 2C, column 3).

To investigate the possibility that the tyrosine phosphorylation status of TbPIP39 might modulate its activity, the activity of TbPIP39 against pNPP was assayed at time points after incubation with FGR kinase to generate the tyrosine-phosphorylated form (Fig. 2D). As a control, the nonphosphorylable TbPIP39 mutant (Y278F) was incubated under the same conditions. TbPIP39 activity was progressively enhanced with increasing phosphory-
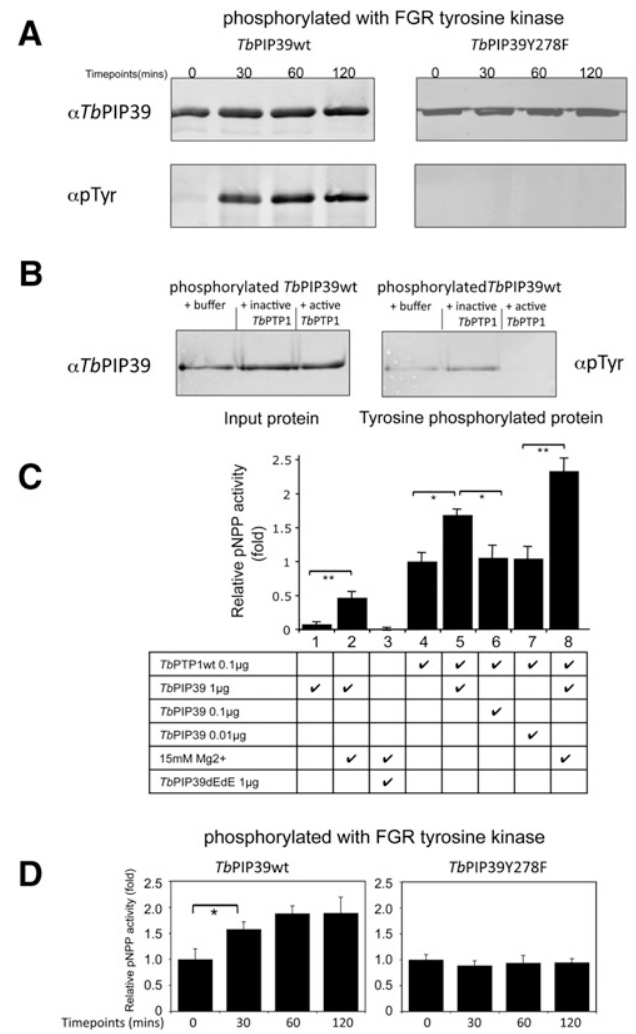

Figure 2. TbPIP39 is a phosphatase with regulatory interaction with TbPTP1. (A). Recombinant TbPIP39 was incubated with FGR kinase to generate a tyrosine-phosphorylated form over the course of $120 \mathrm{~min}$. Tyrosine phosphorylation of the recombinant protein was detected using the phophotyrosine-specific antibody 4G10 ( $\alpha$-pTyr; Millipore). The right panel shows the same analysis using a mutant form of TbPIP39 in which the predicted tyrosine phosphorylation site Y278 is mutated to phenylalanine (F278). In this case, $\alpha$-pTyr reactivity is lost. (B) Tyrosinephosphorylated TbPIP39 is a substrate of TbPTP1. TbPIP39 was incubated with FGR kinase to generate tyrosine-phosphorylated protein, this being detected with the 4G10 antibody ( $\alpha$-pTyr). Upon incubation with wild-type TbPTP1, tyrosine phosphorylation of TbPIP39 is lost. In contrast, when incubated with either buffer alone or a catalytically dead (C229S) TbPTP1 mutant, TbPIP39 remains phosphorylated. $(C)$ Activity against pNPP of recombinant TbPIP39 in the absence (column 1) or presence (column 2) of $\mathrm{Mg}^{2+}$. Column 3 shows the same assay as in column 2, using a dEdE mutant of TbPIP39. Columns 4-7 show the activity against pNPP of TbPTP1 alone (column 4) or in the presence of $1 \mu \mathrm{g}$ (column 5), $0.1 \mu \mathrm{g}$ (column 6), or $0.01 \mu \mathrm{g}$ of $\mathrm{TbPIP} 39$. No $\mathrm{Mg}^{2+}$ was included in the reactions in columns 4-7, preventing TbPIP39 activity. Statistical analyses used a general linear model. $\left(^{\star}\right) P<0.05 ;\left(^{\star \star}\right) P<0.001$. (D) Activity against pNPP of recombinant TbPIP39 as it becomes tyrosinephosphorylated by FGR kinase. With increasing phosphorylation, the activity increases. (Right graphs) In TbPIP39-Y278F, no enhanced activity is observed, demonstrating that tyrosine phosphorylation on Y278 is responsible for TbPIP39 regulation. The input recombinant proteins are those depicted in $A$. Statistical analyses used a general linear model. $\left(^{\star}\right) P<0.05$.

lation, while the nonphosphorylatable mutant showed no enhanced activity above its baseline activity. This confirmed that the activity of TbPIP39 was enhanced upon 
the phosphorylation of $\mathrm{Y} 278$, this site being the target of TbPTP1 activity (Fig. 2B).

As well as TbPTP1 regulating TbPIP39 activity, we also found evidence that TbPIP39 could reciprocally influence the activity of TbPTP1. Thus, although $1 \mu \mathrm{g}$ of TbPIP39 showed negligible activity against $\mathrm{pNPP}$ in the absence of magnesium (Fig. 2C, column 1), when it was incubated together with $0.1 \mu \mathrm{g}$ of TbPTP1, the overall activity of the combined phosphatase enzymes was significantly greater than the activity of $0.1 \mu \mathrm{g}$ of TbPTP1 alone (Fig. 2C, cf. columns $1,4,5)$. Confirming that this was due to the activity of TbPTP1, the same analysis using the inactive D199A mutant of TbPTP1 generated no increase in phosphatase activity (Supplemental Fig. 2). This demonstrated that a positive interaction existed between TbPTP1 and TbPIP39, such that TbPIP39 promoted the activity of TbPTP1 when in significant molar excess.

In a final assay of the interaction between TbPIP39 and TbPTP1, we investigated the effect on their respective phosphatase activities of incubation with citrate, which acts as a trypanosome differentiation trigger. This was prompted by the structural analysis of the DxDxT class phosphatase SCP1, which exhibits a citrate-binding pocket (Kamenski et al. 2004), the required residues for which are conserved in TbPIP39 (Supplemental Fig. 1A). Therefore, we investigated the ability of citrate and the structurally related differentiation trigger cis-aconitate to moderate the activity of either TbPIP39 or TbPTP1, or the two phosphatases in combination (Supplemental Fig. 3A,B, columns 5-8). This demonstrated that both metabolites produced a concentration-dependent reduction of the enhanced phosphatase activity of TbPTP1/TbPIP39 when combined. Eliminating the possibility that divalent cat- ion chelation was responsible for this, citrate or cisaconitate had no effect on either TbPIP39 or TbPTP1 alone (Supplemental Fig. 3A,B, columns 1-4,9-12).

Combined, these experiments demonstrated that TbPIP39 showed less activity when nonphosphorylated; i.e., as the product of TbPTP1 activity, whereas TbPIP39 could activate TbPTP1. Moreover, we found that this regulatory interaction between TbPTP1 and TbPIP39 was abolished by citrate and cis-aconitate, both of which act as trypanosome differentiation stimuli.

\section{Life cycle regulation and glycosomal location of $\mathrm{TbPIP39}$}

TbPTP1 acts in stumpy forms to prevent the parasites undergoing spontaneous differentiation to procyclic forms (Szöőr et al. 2006). To investigate whether its substrate, TbPIP39, was present at the same life cycle stage, the RNA and protein expression of TbPIP39 was investigated in bloodstream slender, bloodstream stumpy, and cultured procyclic forms of $T$. brucei. Although the mRNA for TbPIP39 was abundant in all life cycle stages (albeit enriched in bloodstream stumpy forms) (Fig. 3A), TbPIP39 protein was barely detectable in slender forms, but was expressed in stumpy and elevated during differentiation and in procyclic forms (Fig. 3B; Supplemental Fig. 4A), demonstrating regulation at the level of protein synthesis or turnover. Interestingly, the migration of the TbPIP39 protein was altered between stumpy and procyclic forms (Fig. 3B), there being a slightly higher-molecular-weight form in the latter. This was expected to represent the phosphorylated form of TbPIP39. To establish the tyrosine phosphorylation status of TbPIP39 during differentiation,
A
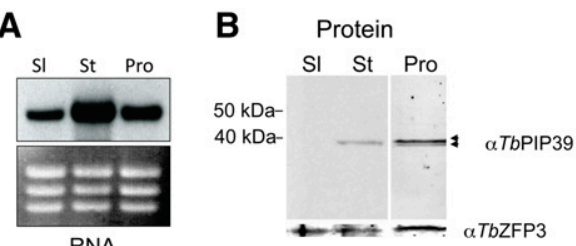

D

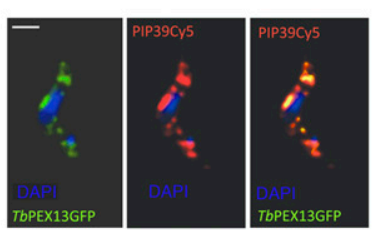

Digitonin/ mg protein

C

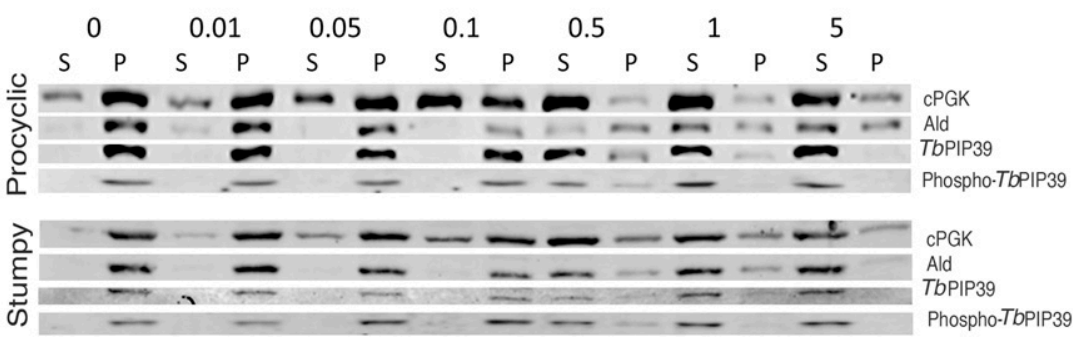

Figure 3. TbPIP39 is a procyclic-enriched glycosomal phosphoprotein. (A) Northern blot of TbPIP39 expression in bloodstream slender $(\mathrm{S} 1)$, bloodstream stumpy $(\mathrm{St})$, or cultured procyclic (Pro) forms. The bottom panel shows the ethidium bromidestained rRNA, indicating loading. $(B)$ Western blot of TbPIP39 in bloodstream slender $(\mathrm{Sl})$, bloodstream stumpy $(\mathrm{St})$, or cultured procyclic (Pro) forms. In stumpy forms, a lower-molecular-weight form is observed, whereas in procyclic forms, a higher-molecular-weight form predominates (arrowheads). The constitutively expressed protein TbZFP3 (Paterou et al. 2006) is included as a loading control. An empty intervening lane between the stumpy and procyclic form samples has been removed for clarity. $(C)$ Digitonin fractionation of procyclic and stumpy form cells reacted with an antibody specific for TbPIP39, TbPIP39 (phospho-Y278), a glycosomal protein (aldolase), or a cytosolic protein (cytosolic PGK). Exposures have been adjusted to best reveal the distribution between fractions for each protein and are not equivalent between the distinct profiles. In each case, the digitonin concentration is shown with the cell extract being separated into either soluble or pelleted (organellar) fractions. TbPIP39 cofractionates with glycosomal aldolase. A quantitative analysis of the fractionation of procyclic forms is shown in Supplemental Figure 6. (D) Localization of TbPIP39 (red) with an N-terminal GFP fusion of the glycosomal protein TbPEX13 (green). The two proteins colocalize precisely. DNA of the cells was counterstained using DAPI (blue). Bar, $5 \mu \mathrm{m}$. 
an anti-peptide tyrosine phospho-specific antibody directed to Y278 of TbPIP39 (ELD HWR TDE $\mathrm{Y}^{\star} \mathrm{TK} \mathrm{C}$ ) was generated. This revealed enhanced phosphorylation of TbPIP39 within 120 min of exposure to cis-aconitate or the tyrosine phosphatase inhibitor BZ3 (Supplemental Fig. 4B). Moreover, reactivity of the phospho-specific antibody was lost upon treatment of procyclic cell extracts with TbPTP1 (Supplemental Fig. 5B C), establishing Y278 as the tyrosine phosphorylation site of TbPIP39 in vivo and the target of TbPTP1. The expression of TbPIP39 was also elevated in stumpy forms exposed to $20^{\circ} \mathrm{C}$ (Supplemental Fig. 4C)-conditions that sensitize trypanosomes to the differentiation stimuli CCA (Engstler and Boshart 2004), and elevate the expression and surface distribution of PAD2, one member of the protein family responsible for conveying the CCA differentiation signal (Dean et al. 2009). Hence, the developmental expression, thermal regulation, and predicted phosphorylation profile of TbPIP39 matched expectations for a TbPTP1 substrate involved in the differentiation control pathway.

When the cellular location of TbPIP39 was investigated by immunofluorescence, a punctate staining was detected, similar to that of trypanosome glycosomal proteins. This was supported by the analysis of the TbPIP39 protein sequence, which exhibited a predicted C-terminal peroxisomal location signal (PTS1) (-SRL) (Supplemental Fig. 1A; Opperdoes and Szikora 2006), and the detection of TbPIP39 in purified procyclic form glycosomes by proteomic analysis (Colasante et al. 2006). Nonetheless, to verify that TbPIP39 associated with the glycosomes, we assessed the fractionation of the protein under a digitonin detergent titration. This demonstrated that TbPIP39 cofractionated with a glycosomal marker protein (aldolase) in both procyclic and stumpy forms, this profile being shared with the phosphorylated form of TbPIP39 detected using the Y278 phospho-specific antibody (Fig. 3C; Supplemental Fig. 6B). Confirming its association with glycosomes, TbPIP39 colocalized with GFP in a transgenic procyclic line expressing an N-terminal GFP fusion of TbPEX13, a glycosomal import protein (Fig. 3D; Verplaetse et al. 2009; the plasmid was a kind gift of P. Michels, Brussels). Hence, by sequence prediction, biochemical and cellular fractionation, and colocalization studies, TbPIP39 was identified as a glycosome-associated protein.

\section{TbPIP39 RNAi inhibits differentiation of bloodstream forms}

To investigate whether TbPIP39 was a component of the differentiation signaling pathway, its mRNA was ablated by tetracycline-regulated RNAi. To assay early differentiation events in a biologically relevant context, it was important to assay stumpy cells capable of synchronous differentiation to procyclic forms. Thus, the T. brucei AnTat1.1 90:13 pleomorphic line was transfected with the stem-loop RNAi vector pALC14 containing opposing fragments of the TbPIP39 gene. A transfectant line was then grown for $6 \mathrm{~d}$ in mice provided either without (-DOX) or with (+DOX) $200 \mu \mathrm{g} / \mathrm{mL}$ doxycyline in their drinking water to induce transcript ablation, and hence prevent TbPIP39 protein expression in developing stumpy forms. Although TbPIP39 was $\sim 85 \%$ depleted in the +DOX parasites (Fig. 4A), no reproducible difference between the progression of the parasitaemia for the uninduced $(-\mathrm{DOX})$ or induced (+DOX) parasites was observed; moreover, both samples generated highly enriched stumpy form populations within $6 \mathrm{~d}$ (Supplemental Fig. 7). This demonstrated that TbPIP39 was not required for stumpy formation or viability. The stumpy forms were then harvested and incubated for $16 \mathrm{~h}$ at either $37^{\circ} \mathrm{C}$ or $20^{\circ} \mathrm{C}$, the latter sensitizing the parasites to physiological levels of CCA (i.e., $\sim 0.1 \mathrm{mM}$ ) (Engstler and Boshart 2004; Dean et al. 2009). Both the +DOX and -DOX populations were then exposed to $0 \mathrm{mM}, 0.1 \mathrm{mM}$, or $6 \mathrm{mM}$ cis-aconitate in vitro and assayed for their expression of the differentiation marker EP procyclin after 4 and $24 \mathrm{~h}$ to detect effects on the initiation of

A

\begin{tabular}{|c|c|c|c|c|c|c|c|}
\hline Time (h) & oh & \multicolumn{3}{|c|}{$4 \mathrm{~h}$} & \multicolumn{3}{|c|}{$24 \mathrm{~h}$} \\
\hline TbPIP39 & - & . & $\because$ & - & • & $m$ & ـ \\
\hline Tub & $=$ & & & & & 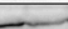 & -1 \\
\hline Dox & \begin{tabular}{l|l}
- & +
\end{tabular} & $-\quad+$ & $-1+$ & $-1+$ & $-1+$ & $-1+$ & $-\quad+$ \\
\hline $\mathrm{CA}(\mathrm{mM})$ & $0 \mathrm{mM}$ & $0 \mathrm{mM}$ & $0.1 \mathrm{mM}$ & $6 \mathrm{mM}$ & $0 \mathrm{mM}$ & $0.1 \mathrm{mM}$ & $6 \mathrm{mM}$ \\
\hline
\end{tabular}

B

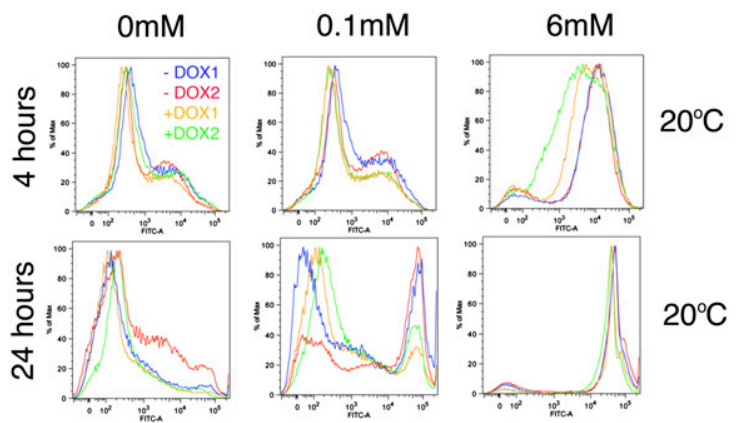

Figure 4. TbPIP39 depletion in stumpy forms inhibits differentiation. (A) Western blots of TbPIP39 from samples derived from TbPIP39-RNAi cells either induced (+Dox) or uninduced (-Dox) with doxycycline. Samples were incubated overnight at $20^{\circ} \mathrm{C}$, and then proteins were isolated $0 \mathrm{~h}, 4 \mathrm{~h}$, or $24 \mathrm{~h}$ after exposure to $0 \mathrm{mM}, 0.1 \mathrm{mM}$, or $6 \mathrm{mM}$ cis-aconitate. The bottom panels represent analysis of the same samples using an antibody against $\alpha$-tubulin as a loading control. (B) Flow cytometry traces of EP procyclin expression in four independent stumpy cell populations either induced (+Dox, orange and green traces) or not (-Dox, blue and red traces) to ablate TbPIP39 by RNAi. Individual stumpy cell samples were derived from day 6 infections of mice either with or without doxycyline in their drinking water. Samples were incubated at $20^{\circ} \mathrm{C}$ before the addition of cis-aconitate, and flow cytometry was carried out either $4 \mathrm{~h}$ or $24 \mathrm{~h}$ later. The induced populations showed reduced differentiation at $0.1 \mathrm{mM}(4 \mathrm{~h}$ and $24 \mathrm{~h})$; at $6 \mathrm{mM}$, differentiation was efficient in all populations (likely enabled by the remaining $\sim 15 \%$ TbPIP39 in the RNAi line), although it was delayed in the induced samples. Flow cytometry traces from this experiment with cells incubated at $37^{\circ} \mathrm{C}$ are available in Supplemental Figure 8. 
differentiation. Although the extent of TbPIP39 depletion and the overall kinetics of EP procyclin expression in different experiments varied, perhaps reflecting the amount of remaining enzyme following RNAi $(\sim 15 \%)$, the TbPIP39-depleted parasites showed reduced differentiation with $0.1 \mathrm{mM}$ cis-aconitate (mean, $58 \%$ reduction; range, $52 \%-67 \%$ reduction at $24 \mathrm{~h}$ ) (Fig. $4 \mathrm{~B}$ ), this effect being consistent in five out of five experiments, whereas at $6 \mathrm{mM}$ cis-aconitate, an effect of TbPIP39 depletion was observed in only two out of five experiments. Hence, the depletion of TbPIP39 reduced the differentiation of stumpy forms, with a reduction of sensitivity to physiological levels of CCA. Being detected in uniform populations of cells before the onset of outgrowth as procyclic forms, we conclude that TbPIP39 acts as a positive signaling component operating downstream from, and negatively regulated by, TbPTP1.

To confirm that the differentiation signaling response required the glycosomal location of TbPIP39, we investigated the ability of mutants with a deleted or an epitope tag-blocked C-terminal PTS1 signal to rescue the TbPIP39 RNAi-mediated differentiation phenotype. To achieve this, monomorphic bloodstream forms capable of TbPIP39 RNAi (Supplemental Fig. 9) were engineered to express recoded synthetic genes that would be immune to RNAi, but encode proteins of the same amino sequence as endogenous TbPIP39 (Supplemental Fig. 10A,B). Initially, the monomorphic TbPIP39 RNAi line was stimulated to differentiate with cis-aconitate, demonstrating reduced procyclin expression over $48 \mathrm{~h}$ when RNAi was induced with tetracycline (Fig. 5A). When a wild-type recoded TbPIP39 copy was coexpressed in the same line, however, differentiation was fully restored, supporting rescue of the phenotype (Fig. 5B). In contrast, the expression of TbPIP39ASRL or TbPIP39-C terminal Ty, both of which were cytosolic by digitonin fractionation (Supplemental Fig. 10C), failed to rescue the differentiation phenotype (Fig. 5C,D), as did a catalytically inactive (TbPIP39dEdE) TbPIP39 mutant (Fig. 5E). These observations support the glycosomal location and activity of TbPIP39 being important for differentiation signaling.

\section{Discussion}

We demonstrated previously that the tyrosine phosphatase TbPTP1 acts as a "molecular brake" that prevents differentiation of bloodstreams stumpy forms in the mammalian bloodstream (Szöör et al. 2006). In this study, we identify a downstream substrate of TbPTP1 as a second phosphatase, TbPIP39. TbPIP39 is shown to be a developmentally regulated magnesium-dependent phosphatase whose activity is modulated by tyrosine phosphorylation. Moreover, we show that TbPIP39 contributes to the efficient differentiation to procyclic forms, thereby invoking the existence of a phosphatase signaling cascade regulating trypanosome development. Supporting this, we show regulatory cross-talk between TbPIP39 and TbPTP1 in vitro, this being abolished by the differentiation regulators citrate and cis-aconitate. Finally, we demonstrate that TbPIP39 is glycosomal, identifying this
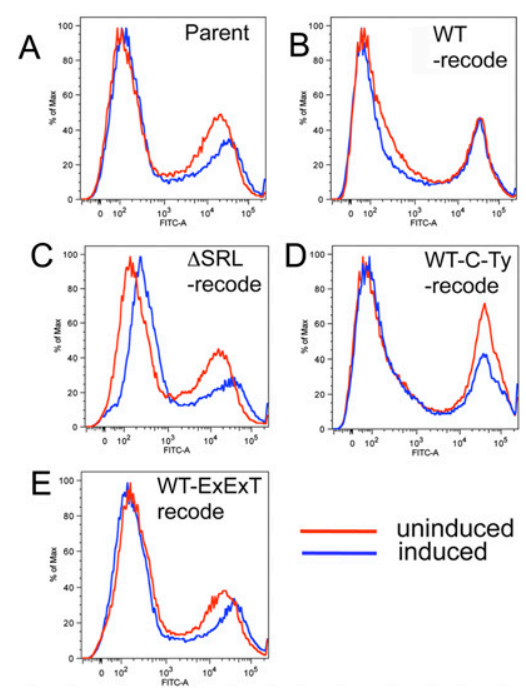

Figure 5. An intact glycosomal targeting signal is required for TbPIP39-dependent differentiation. (A) T. brucei single-marker bloodstream forms were transfected with the TbPIP39 RNAi construct used in Figure 4. The resulting transfectant cell line was then induced (blue) or not induced (red) to ablate TbPIP39 by incubation with $1 \mu \mathrm{g} / \mathrm{mL}$ tetracycline for $2 \mathrm{~d}$. The RNAidepleted cells and the uninduced controls were then stimulated to differentiate to procyclic forms by the addition of $6 \mathrm{mM}$ cisaconitate and the expression of the differentiation marker EP procyclin monitored by flow cytometry. Depletion of TbPIP39 resulted in reduced differentiation efficiency at $48 \mathrm{~h}$ after exposure to cis-aconitate. $(B)$ The same cell line as in $A$ was transfected with pHD451 expressing an intact recoded synthetic TbPIP39 gene, expressed under tetracycline regulation. Cells were grown with tetracycline for $2 \mathrm{~d}$, allowing silencing of the endogenous TbPIP39 transcript and expression of the recoded gene. Differentiation was then monitored for EP procyclin expression $48 \mathrm{~h}$ after exposure to $6 \mathrm{mM}$ cis-aconitate using flow cytometry. The differentiation phenotype was fully rescued. $(C)$ As in $B$, but the recoded synthetic gene product lacked the $\triangle$ SRL glycosomal targeting signal and redistributed to the cytosolic fraction (Supplemental Fig. 10C). In this case, the differentiation defect was not rescued. $(D)$ As in $B$, but the recoded synthetic gene product contained a Tyl epitope tag after the $\Delta$ SRL glycosomal targeting signal. As with the truncated mutant, the protein was redistributed to the cytosolic fraction (Supplemental Fig. 10C) and the differentiation defect was not rescued. (E) As in $B$, but the recoded synthetic gene product was mutated for the DxDxT motif (to ExExT) to prevent catalytic activity. No rescue of the differentiation defect was observed.

molecule as a new marker for distinguishing bloodstream from procyclic form glycosomes, and the first example of a signaling molecule targeted to this fundamental metabolic organelle in trypanosomes. To our knowledge, this is the first well-characterized signaling pathway targeted directly to a peroxisomal-type organelle in any eukaryotic cell.

TbPIP39 was identified as an unusual type of Ser/Thr phosphatase, a DxDxT phosphatase. This is an emerging family of phosphatases, and one of a group of such phosphatases encoded in kinetoplastid genomes $(14,13$, and 13 members, in T.brucei, T.cruzi, and L. major, respectively) 
(Brenchley et al. 2007), but the only representative possessing a predicted PTS1. Consistent with expectations for this class of molecules (Selengut and Levine 2000; Ndubuisil et al. 2002), TbPIP39 showed magnesiumdependent activity, with its activity being abolished by mutation of the predicted magnesium-coordinating aspartate residues in the DxDxT phosphoryl acceptor motif (Collet et al. 1998). Interestingly, however, when TbPIP39 was coincubated with TbPTP1, the overall phosphatase activity of the combined enzymes was elevated over their predicted additive values. Since the effect was observed with inactive TbPIP39 (i.e., in the absence of magnesium) and was not recapitulated when TbPIP39 was incubated with a catalytically dead C229S mutant of TbPTP1, we assign the enhanced phosphatase activity of TbPTP1/ TbPIP39 to an activation of TbPTP1. While being potentially muted by the relatively high levels of citrate in the Escherichia coli expression system $(\sim 5 \mathrm{mM})$, we observed that the activation of recombinant TbPTP1 required a 10-fold excess of its substrate, TbPIP39, in vitro. In vivo, the physiological interactions between the proteins could be enhanced significantly by their relative context or the presence of associated proteins in the cell, but nonetheless TbPIP39 is in considerable excess of TbPTP1 in stumpy forms (Supplemental Fig. 4D). Hence, TbPIP39 is predicted to promote its own dephosphorylation by activating TbPTP1. Given our finding that the Y278-phosphorylated form of TbPIP39 shows greater phosphatase activity than its unphosphorylated form, these observations combine to generate a feedback loop whereby TbPIP39 reinforces its own dephosphorylation, and hence repression, by activating TbPTP1.

Although TbPTP1 was found to be a cytosolic/ cytoskeletally associated protein (Szöőr et al. 2006), its substrate, TbPIP39, was found to be glycosomally associated. This apparent paradox can be resolved by considering the maturation of proteins as they are trafficked to peroxisome-related organelles, such as glycosomes. Such proteins are initially translated in the cytosol and then folded. The peroxisomal targeting signal is then recognized, and the protein is translocated to the peroxisome membrane where it is imported via peroxins to the lumen. This passage through the cytosol, from synthesis to import, has been measured to take from 5 to $60 \mathrm{~min}$ (McNew and Goodman 1994; Terlecky et al. 2001), providing ample opportunity for TbPIP39 to be phosphorylated or dephosphorylated prior to glycosome targeting.

Based on our experiments, we propose a new model for the early steps in trypanosome differentiation (Fig. 6). In stumpy forms, TbPIP39 would be synthesized, but held relatively inactive through its dephosphorylation by TbPTP1, this being reinforced by the enhanced activity of TbPTP1 generated by its substrate when in excess. When trafficked to the glycosome, dephosphorylated, inactive TbPIP39 would not stimulate differentiation. When entering the tsetse, however, temperature reduction to $20^{\circ} \mathrm{C}$ would facilitate CCA transport into the cell via PAD expression (Dean et al. 2009). If matching our in vitro observations, this would prevent the feedback activation of TbPTP1 by TbPIP39, although CCA may

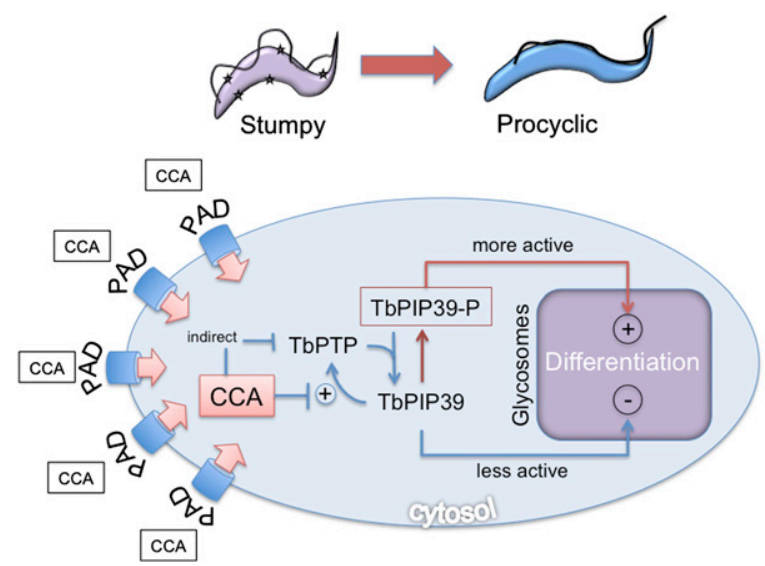

Figure 6. A model depicting the regulatory interactions between TbPTP1 and TbPIP39 when exposed to the differentiation triggers citrate or cis-aconitate. The CCA signal is conveyed by PAD proteins expressed on the surface of stumpy forms. This inhibits the activation of TbPTP1 by TbPIP39, although TbPTP1 may also be inactivated indirectly. Upon the inactivation of TbPTP1, phosphorylated TbPIP39 predominates, this being generated by an unidentified protein kinase. TbPIP39, activated by phosphorylation, is directed toward the glycosomes by its C-terminal PTS1 signal, and therein promotes a differentiation response.

also inhibit TbPTP1 indirectly through other unknown mechanisms. Combined with the enhanced expression of TbPIP39 at low temperature, this would increase the level of phosphorylated TbPIP39, which, upon entry to the glycosome, would promote differentiation.

The proposed pathway is reminiscent of cell fate decision pathways in other eukaryotes, whereby phosphorylation-dephosphorylation events coordinate a transition from one stable state to another-a so-called bistable switch (Pomerening et al. 2003; Ingolia and Murray 2007). In this case, the activation of TbPTP1 by TbPIP39 would repress differentiation by enhancing the dephosphorylation of TbPIP39, whereas exposure to CCA would alleviate this repression, favor TbPIP39 phosphorylation, and so stimulate differentiation. Importantly, the sequestration of phosphorylated TbPIP39 in the glycosomal compartment would protect it from further TbPTP1 activity, rendering its activation irreversible. Although metabolic regulation via phosphorylation represents an obvious point of downstream control (Christofk et al. 2008) and is a known regulator of differentiation events (Milne et al. 1998; Morris et al. 2002), glycosomes are predicted to comprise $>200$ distinct proteins (Colasante et al. 2006; Opperdoes and Szikora 2006) associated with ether lipid biosynthesis, the $\beta$ oxidation of fatty acids, purine salvage, pyrimidine biosynthesis, gluconeogenesis, and isoprenoid biosynthesis, in addition to glycolysis. Several identified DxDxT phosphatases, including Dullard, a regulator of mammalian nuclear membrane biogenesis, exhibit lipid phosphatase activity (Kim et al. 2007; Reddy et al. 2008), suggesting that these enzymes may have the potential to regulate second messenger signaling pathways. Hence, any one, or several, of the trypanosome 
glycosomal activities may be regulated by TbPIP39 with consequences independent of, or indirectly linked to (Chambers et al. 2008), metabolism.

The existence of signaling pathways directed to peroxisome-like organelles, and specifically glycosomes, has been proposed previously (Albert et al. 2005; Michels and Rigden 2006). Our experiments establish that such a pathway exists, comprising a phosphatase signaling cascade that drives an early step in cell type differentiation in African trypanosomes. Moreover, the bistable regulation of this pathway by the physiological conditions encountered during stumpy form transmission suggests a new elegance to trypanosome developmental control, linked to the biology of their key metabolic organelle, the glycosome.

\section{Materials and methods}

\section{Parasite growth and transfection}

Bloodstream form and procyclic form trypanosomes were cultured in vitro in HMI-9 medium (Hirumi and Hirumi 1984) or SDM-79 medium (Brun and Schonenberger 1979), respectively.

Culturing, differentiation, pleomorphic trasnfection, and coldshock assays of parasites were performed as described in Dean et al. (2009) using the T. brucei AnTat1.1 90:13 cell line (Engstler and Boshart 2004), transfectants being generated using an AMAXA nucleofector protocol (T-cell nucleofection buffer, program X001) and selected using $0.5 \mathrm{mg} \mathrm{mL}^{-1}$ puromycin. Stumpyenriched populations were obtained by DEAE cellulose purification (Lanham 1968) of parasites 6-7 d after infection into cyclophosphamide-treated mice.

For ectopic expression, T. brucei Lister 427 bloodstream forms were used, these being engineered previously to express the tetracycline repressor protein (Wirtz et al. 1999), enabling regulated gene expression of wild-type or substrate-trapping forms of TbPTP1 (Szöör et al. 2006). Established, cultured procyclic forms were T. brucei Lister 427.

A transgenic procyclic line expressing an $\mathrm{N}$-terminal GFP fusion of PEX13, a glycosomal import protein, was created by transfection of a previously characterized expression plasmid kindly provided by Paul Michels (Université Catholique de Louvain, Brussels) (Verplaetse et al. 2009).

\section{DNA cloning}

The TbPIP39 coding region was amplified using TbPIP39-specific primers Primer 1 (see Table 1) and Primer 2, and was integrated into the pGEX4T1 (GE Heathcare Lifesciences) protein expression vector for recombinant protein production. Primer 3 and Primer 4 (see Table 1) were used to insert TbPIP39 into the
pHD451 trypanosome ectopic expression vector (Biebinger et al. 1997). The pGEX4T1TbPIP39 construct was used as a template for site-directed mutagenesis in order to mutate TbPIP39 Tyr 278 to phenylalanine (abbreviated Y278F TbPIP39). This was carried out using a commercial site-directed mutagenesis kit (Stratagene) using the mutagenesis primers Primer 5 and Primer 6 (see Table 1). Recoded synthetic genes (Supplemental Fig. 10) were synthesized by Geneart (http://www.geneart.com), recloned into pHD451, and transfected into monomorphic cells already containing the pALC14TbPIP39 RNAi plasmid as a stable integrant.

\section{Generation of TbPIP39 RNAi cell line}

The TbPIP39 reading frame was amplified from genomic DNA by PCR using Primer 7 and Primer 8 (Table 1), and was integrated into the HindIII-XbaI and XhoI-NdeI cloning sites of pALC14 (Pusnik et al. 2007) to generate the pALC14TbPIP39 RNAi plasmid.

\section{Antibody production}

Polyclonal antibody was produced in rabbits against full-length recombinant TbPIP39 by Eurogentec; an anti-peptide phospho278 YTbPIP39 antibody was raised in rabbits against the sequence N-ELDHWRTDE-[phospho]Y-TKC-C from the amino acid sequence at position 269-281 in TbPIP39 (Eurogentec). The resulting antibody was affinity-purified against the phosphorylated peptide immunogen, and was counterselected against the nonphosphorylated peptide to maximize specificity.

\section{Substrate trapping with $\mathrm{Tb} P T P 1 D 199 A$}

The production and purification of the substrate-trapping mutant of TbPTP1 (TbPTP1D199A) was described in Szoor et al. (2006). Purified His-TbPTP1D199A was mixed with His chelating Ni-NTA Agarose beads (Qiagen) for $30 \mathrm{~min}$ at $4^{\circ} \mathrm{C}$. The beads were then collected by centrifugation and washed three times with ice-cold His purification lysis buffer $(20 \mathrm{mM}$ Tris, $250 \mathrm{mM}$ $\mathrm{NaCl}, 1 \%$ Triton-X, $1 \mathrm{mM} \beta$ mercaptoethanol, $5 \mathrm{mM}$ Imidazole at $\mathrm{pH}$ 7.5-8) supplemented with complete, and EDTA-free protease inhibitor cocktail (Roche).

Approximately $5 \times 10^{8}$ DEAE-purified T. brucei stumpy cells were harvested by centrifugation, and the cell pellet was frozen in liquid nitrogen. Cells were then lysed in the presence of $150 \mathrm{mM}$ PTP1B inhibitor BZ3 (Calbiochem) by addition of $500 \mu \mathrm{L}$ of His purification lysis buffer supplemented with complete, and EDTA-free protease inhibitor cocktail (Roche). The extract was lysed by two freeze/thaw cycles at $-80^{\circ} \mathrm{C}$, centrifuged for $20 \mathrm{~min}$ at $4^{\circ} \mathrm{C}$ in a microfuge, and then sonicated for $3 \mathrm{~min}$ in a water bath. Quantification of cellular protein was performed by the Bradford method following the manufacturer's instructions.

Table 1. Oligonucleotides used in the study

\begin{tabular}{ll}
\hline Oligonucleotide name & \multicolumn{1}{c}{ Sequence } \\
\hline Primer 1 (BamHI Fwd) & 5'-TATGGATCCATGGTGAGGACGACACGCTTTTC-3' $^{\prime}$ \\
Primer 2 (XhoI Rev) & 5'-ATACTCGAGCTAAAGTCTTGAAGGAGTGTGTC-3' $^{\prime}$ \\
Primer 3 (HindIII Fwd) & 5'-TATAAGCTTATGGTGAGGACACGCTTTTC-3' $^{\prime}$ \\
Primer 4 (BamHI Rev) & 5'-ATAGGATCCCTAAAGTCTTGAAGGAGTGTGTC-3' $^{\prime}$ \\
Primer 5 (Y278F Fwd) & 5'-CCATTGGCGTACGGATGAGTTCACAAAGTGTGACGACTTTCG-3' \\
Primer 6 (Y278F Rev) & 5'-CGAAAGTCGTCACACTTTGTGAACTCATCCGTACGCCAATGG-3' \\
Primer 7 (HindIIIBamHIFwd) & 5'-ATAAAGCTTGGATCCGTGAGGACGACACGCTTTTCACG-3' \\
Primer 8 (XhoINdeI Rev) & 5'-TGCCATATGCTCGAGGGGAGTTGTAACACCTGCACCTCC-3' \\
\hline
\end{tabular}


Approximately $0.5 \mathrm{mg}$ of fresh cell extract was incubated for $1.5 \mathrm{~h}$ on ice with $20-30 \mu \mathrm{L}$ of Ni-NTA beads coated with Histagged protein; nonspecific interactions were removed by washing the beads three times with $1 \mathrm{~mL}$ of $20 \mathrm{mM}$ Tris, $250 \mathrm{mM}$ $\mathrm{NaCl}$, and $20 \mathrm{mM}$ imidazole (pH: 8.0), and the bound proteins were eluted by addition of SDS-PAGE sample buffer.

\section{Mass spectrometry}

Eluate from substrate-trapping columns was separated by SDSPAGE, and Coomassie Blue-stained bands were excised and washed in $100 \mathrm{mM}$ ammonium bicarbonate with shaking for $1 \mathrm{~h}$ at room temperature, followed by a second wash in $50 \%$ acetonitrile/100 mM ammonium bicarbonate. Proteins were reduced with $3 \mathrm{mM}$ DTT in $100 \mathrm{mM}$ ammonium bicarbonate for $30 \mathrm{~min}$ at $60^{\circ} \mathrm{C}$, followed by alkylation with $10 \mathrm{mM}$ iodoacetamide for $30 \mathrm{~min}$ in the dark at room temperature. The gel pieces were washed with $50 \%$ acetonitrile/100 mM ammonium bicarbonate, shaking for $1 \mathrm{~h}$ at room temperature, and then were dehydrated by incubation with $0.1 \mathrm{~mL}$ of acetonitrile for $10 \mathrm{~min}$ at room temperature. All liquid was removed and gel pieces were dried to completion under vacuum, then rehydrated with a sufficient volume of trypsin (Promega sequencing grade, $2 \mathrm{mg} / \mathrm{mL}$ in $25 \mathrm{mM}$ ammonium bicarbonate) to cover the gel pieces. Digestion was performed overnight at $37^{\circ} \mathrm{C}$, the gel pieces washed for $10 \mathrm{~min}$ with $0.02 \mathrm{~mL}$ of $1 \%$ formic acid, and then $0.02 \mathrm{~mL}$ acetonitrile was added. After $10 \mathrm{~min}$ of incubation, all liquid was transferred to a fresh tube, and the tryptic peptides were dried to completion.

Tryptic peptides were solubilized in $0.5 \%$ formic acid and $2 \%$ acetonitrile, and were fractionated on a nanoflow HPLC system (Famos/Switchos/Ultimate, LC Packings) before being analyzed by electrospray ionization (ESI) mass spectrometry on a Q-STAR Pulsar i hybrid MS/MS System. Peptide separation was performed on a Pepmap C18 reversed-phase column (LC Packings) using a $5 \%-85 \% \mathrm{v} / \mathrm{v}$ acetonitrile gradient (in $0.5 \% \mathrm{v} / \mathrm{v}$ formic acid) run over $45 \mathrm{~min}$. The flow rate was maintained at $0.2 \mu \mathrm{L}$ per minute. Mass spectrometric analysis was performed using a 3-sec survey MS scan, followed by up to four MS/MS analyses of the most abundant peptides (3 sec per peak) in InformationDependent Acquisition (IDA) mode, choosing 2+ to 4+ ions above threshold of 30 counts, with dynamic exclusion for $120 \mathrm{sec}$.

Mass spectrometry data was analyzed using Applied Biosystems Analyst QS (version 1.1) software and the automated Matrix Science Mascot Daemon server (version 2.1.06). Protein identifications were assigned using the Mascot search engine, with carbamidomethylation of cysteines and variable methionine oxidation being allowed. An MS tolerance of 1.2 Da for MS and $0.4 \mathrm{Da}$ for MS/MS analysis was used. Searches were performed against the T. brucei genome database (version 3) obtained from the Wellcome Trust Sanger Institute and maintained on a local Mascot server.

\section{Digitonin permeabilization assay}

Approximately $5 \times 10^{8}$ procyclic forms were collected to perform digitonin permeabilization. The cell suspension was diluted to $\sim 1 \mathrm{mg}$ of total protein per milliliter, and increasing amounts of digitonin (0.01-5 mg/mg total protein) were used in digitonin assays according to Ferella et al. (2008).

\section{Immunoprecipitation}

Transgenic bloodstream form cell lines in which either wild-type TbPTP1 or TbPTP1-D199A proteins were expressed ectopically with a C-terminal Tyl epitope tag sequence (Szöör et al. 2006) were treated with $6 \mathrm{mM}$ cis-aconitate for $24 \mathrm{~h}$. Immunoprecipitation was carried out using $1 \times 10^{8}$ to $5 \times 10^{8}$ cells as described in Paterou et al. (2006).

\section{Western and Northern blotting}

Western blotting and Northern blotting was performed as described in Tasker et al. (2000). For Western blotting, primary antibodies were diluted 1:1000 and secondary antibodies were diluted 1:5000. Proteins were detected using the LI-COR Odyssey system for quantification against a tubulin or loading control as described in Dean et al. (2009). For Northern blots, a digoxygenin-labeled TbPIP39-specific riboprobe (Roche) was used, hybridization being detected using CDP-star as a reaction substrate.

\section{Phosphatase and kinase activity assays}

Phosphatase activity was measured by monitoring the TbPIP39catalyzed and TbPTP1-catalyzed $(0.01-1 \mu \mathrm{g})$ hydrolysis of pNPP to $p$-nitrophenol (Szöör et al. 2006). Phosphorylation of recombinant TbPIP39 by FGR kinase was performed according to the manufacturer's instructions (Calbiochem). The reaction, containing purified TbPIP39 $(5 \mu \mathrm{g})$, was initiated with the addition of FGR kinase, and samples were taken at time points after incubation $(0,30,60,90,120,240 \mathrm{~min})$ at $30^{\circ} \mathrm{C}$ for Western blots. After the 240-min time point, the remainder of the phosphorylation mixture was loaded on a GSTrap HP column (GE Healthcare Life Sciences) to remove the GST-tagged FGR kinase. After collection of the flow-through from the column containing the phosphorylated TbPIP39, the sample was concentrated on Vivaspin 2 columns with a 10-kDa-molecular-weight cutoff (Sartorius AG) before use in the in vitro TbPTP1 phosphatase assay.

\section{In vitro $\mathrm{Tb} P \mathrm{TP} 1$ phosphatase assay}

To monitor phosphatase activity of phosphorylated TbPIP39, the concentrated phosphorylated TbPIP39 was treated with either $1 \mu \mathrm{g}$ of active wild-type TbPTP1 or the same amount of inactive C229S TbPTP1 mutant in a $100-\mu \mathrm{L}$ phosphatase assay $(50 \mathrm{mM}$ Tris, $50 \mathrm{mM}$ Bis Tris, $100 \mathrm{mM} \mathrm{Na}$ acetate, $1 \mathrm{mM}$ DTT at $\mathrm{pH}$ 5.5). The phosphatase reaction was started with the addition of TbPTP1, and, after $30 \mathrm{~min}$ of incubation at $37^{\circ} \mathrm{C}, 20-\mu \mathrm{L}$ samples were removed for Western blot.

\section{Flow cytometry and immunofluorescence}

Differentiating cells $\left(2 \times 10^{6}\right.$ to $\left.5 \times 10^{6}\right)$ were harvested and fixed in $2 \%$ formaldehyde $/ 0.05 \%$ glutaraldehyde for a minimum of $1 \mathrm{~h}$ at $4^{\circ} \mathrm{C}$. Antibody staining was executed according to Dean et al. (2009). Flow cytometry analysis was performed using the BD LSRII Flow cytometer (Becton Dickenson), and flow cytometry data was analyzed using FlowJO 8.8.6 software (Tree Star, Inc.) with unstained cells; cells stained with only the secondary antibody provided negative controls. For immunofluorescence, air-dried smears of parasites were prepared and fixed in methanol for at least $30 \mathrm{~min}$ at $-20^{\circ} \mathrm{C}$. Cells were rehydrated in PBS for $30 \mathrm{~min}$ before labeling, as described in Dean et al. (2009).

\section{Image acquisition equipment and settings}

Phase-contrast and immunofluorescence microscopy images (Supplemental Fig. 7A) were captured on a Zeiss Axioskop2 (Carl Zeiss Microimaging) with a Prior Lumen 200 light source using a QImaging Retiga 2000RCCD camera; objectives were either Plan Neofluar $\times 63$ (1.25 NA) or Plan Neofluar $\times 100$ (1.30 NA). 
Images were captured via QImage (QImaging). Confocal imaging (Fig. 3D) used a Leica SP5 confocal laser scanning microscope, using $\times 63$ oil immersion objective $(\mathrm{NA}=1.4)$, with 4.2 zoom. The green channel was imaged using a $488-\mathrm{nm}$ argon laser, and the red channel was imaged using a 543-nm helium/neon laser. The final image was acquired using Volocity Software (Improvision Ltd.) version 4.4.

\section{Acknowledgments}

We thank Achim Schnaufer for comments on the manuscript and for suggesting the use of recoded synthetic genes, Paul Michels for TbPEX13-GFP construct, and Michael Boshart and Markus Engstler for the gift of the T. brucei AnTat1.1 90:13 line. We also thank Martin Waterfall for assistance with FACS analysis. This work was supported by grants from the Wellcome Trust, the BBSRC (for the provision of confocal facilities), and by a Strategic Award from the Wellcome Trust for the Centre for Immunity, Infection, and Evolution.

\section{References}

Albert MA, Haanstra JR, Hannaert V, Van Roy J, Opperdoes FR, Bakker BM, Michels PA. 2005. Experimental and in silico analyses of glycolytic flux control in bloodstream form Trypanosoma brucei. J Biol Chem 280: 28306-28315.

Bakker BM, Mensonides FI, Teusink B, van Hoek P, Michels PA, Westerhoff HV. 2000. Compartmentation protects trypanosomes from the dangerous design of glycolysis. Proc Natl Acad Sci 97: 2087-2092.

Barrett MP, Burchmore RJ, Stich A, Lazzari JO, Frasch AC, Cazzulo JJ, Krishna S. 2003. The trypanosomiases. Lancet 362: 1469-1480.

Bastin P, Bagherzadeh Z, Matthews KR, Gull K. 1996. A novel epitope tag system to study protein targeting and organelle biogenesis in Trypanosoma brucei. Mol Biochem Parasitol 77: 235-239.

Biebinger S, Wirtz LE, Lorenz P, Clayton C. 1997. Vectors for inducible expression of toxic gene products in bloodstream and procyclic Trypanosoma brucei. Mol Biochem Parasitol 85: 99-112.

Blanchetot C, Chagnon M, Dubé N, Hallé M, Tremblay ML. 2005. Substrate-trapping techniques in the identification of cellular PTP targets. Methods 35: 44-53.

Brenchley R, Tariq H, McElhinney H, Szoor B, Huxley-Jones J, Stevens R, Matthews K, Tabernero L. 2007. The TriTryp phosphatome: Analysis of the protein phosphatase catalytic domains. BMC Genomics 8: 434. doi: 10.1186/1471-2164-8434.

Broadhead R, Dawe HR, Farr H, Griffiths S, Hart SR, Portman N, Shaw MK, Ginger ML, Gaskell SJ, McKean PG, et al. 2006. Flagellar motility is required for the viability of the bloodstream trypanosome. Nature 440: 224-227.

Brun R, Schonenberger. 1979. Cultivation and in vitro cloning or procyclic culture forms of Trypanosoma brucei in a semidefined medium. Short communication. Acta Trop 36: 289292.

Chambers JW, Kearns MT, Morris MT, Morris JC. 2008. Assembly of heterohexameric trypanosome hexokinases reveals that hexokinase 2 is a regulable enzyme. I Biol Chem 283: 14963-14970.

Christofk HR, Vander Heiden MG, Wu N, Asara JM, Cantley LC. 2008. Pyruvate kinase M2 is a phosphotyrosine-binding protein. Nature 452: 181-186.

Colasante C, Ellis M, Ruppert T, Voncken F. 2006. Comparative proteomics of glycosomes from bloodstream form and pro- cyclic culture form Trypanosoma brucei brucei. Proteomics 6: 3275-3293.

Collet JF, Stroobant V, Pirard M, Delpierre G, Van Schaftingen E. 1998. A new class of phosphotransferases phosphorylated on an aspartate residue in an amino-terminal DXDX $(\mathrm{T} / \mathrm{V})$ motif. J Biol Chem 273: 14107-14112.

Czichos J, Nonnengaesser C, Overath P. 1986. Trypanosoma brucei: Cis-aconitate and temperature reduction as triggers of synchronous transformation of bloodstream to procyclic trypomastigotes in vitro. Exp Parasitol 62: 283-291.

Dean SD, Marchetti R, Kirk K, Matthews K. 2009. A surface transporter family conveys the trypanosome differentiation signal. Nature 459: 213-217.

Engstler M, Boshart M. 2004. Cold shock and regulation of surface protein trafficking convey sensitization to inducers of stage differentiation in Trypanosoma brucei. Genes Dev 18: $2798-2811$.

Fenn K, Matthews KR. 2007. The cell biology of Trypanosoma brucei differentiation. Curr Opin Microbiol 10: 539-546.

Ferella M, Li ZH, Andersson B, Docampo R. 2008. Farnesyl diphosphate synthase localizes to the cytoplasm of Trypanosoma cruzi and T. brucei. Exp Parasitol 119: 308-312.

Hannaert V, Saavedra E, Duffieux F, Szikora JP, Rigden DJ, Michels PA, Opperdoes FR. 2003. Plant-like traits associated with metabolism of Trypanosoma parasites. Proc Natl Acad Sci 100: 1067-1071.

He CY. 2007. Golgi biogenesis in simple eukaryotes. Cell Microbiol 9: 566-572.

He CY, Ho HH, Malsam J, Chalouni C, West CM, Ullu E, Toomre D, Warren G. 2004. Golgi duplication in Trypanosoma brucei. J Cell Biol 165: 313-321.

He CY, Pypaert M, Warren G. 2005. Golgi duplication in Trypanosoma brucei requires Centrin2. Science 310: 11961198.

Hirumi H, Hirumi K. 1984. Continuous cultivation of animalinfective bloodstream forms of an East African Trypanosoma congolense stock. Ann Trop Med Parasitol 78: 327-330.

Ingolia NT, Murray AW. 2007. Positive-feedback loops as a flexible biological module. Curr Biol 17: 668-677.

Kamenski T, Heilmeier S, Meinhart A, Cramer P. 2004. Structure and mechanism of RNA polymerase II CTD phosphatases. Mol Cell 15: 399-407.

Kim Y, Gentry MS, Harris TE, Wiley SE, Lawrence JC Jr, Dixon JE. 2007. A conserved phosphatase cascade that regulates nuclear membrane biogenesis. Proc Natl Acad Sci 104: 6596-6601.

Lanham SM. 1968. Separation of trypanosomes from the blood of infected rats and mice by anion-exchangers. Nature 218: 1273-1274.

Matthews KR. 2005. The developmental cell biology of Trypanosoma brucei. J Cell Sci 118: 283-290.

McNew JA, Goodman JM. 1994. An oligomeric protein is imported into peroxisomes in vivo. J Cell Biol 127: 1245-1257.

Michels PA, Rigden DJ. 2006. Evolutionary analysis of fructose 2,6-bisphosphate metabolism. IUBMB Life 58: 133-141.

Michels PA, Bringaud F, Herman M, Hannaert V. 2006. Metabolic functions of glycosomes in Trypanosomatids. Biochim Biophys Acta 1763: 1463-1477.

Milne KG, Prescott AR, Ferguson MA. 1998. Transformation of monomorphic Trypanosoma brucei bloodstream form trypomastigotes into procyclic forms at 37 degrees $\mathrm{C}$ by removing glucose from the culture medium. Mol Biochem Parasitol 94: 99-112.

Morris JC, Wang Z, Drew ME, Englund PT. 2002. Glycolysis modulates trypanosome glycoprotein expression as revealed by an RNAi library. EMBO I 21: 4429-4438. 
Ndubuisil MI, Kwok BH, Vervoort J, Koh BD, Elofsson M, Crews CM. 2002. Characterization of a novel mammalian phosphatase having sequence similarity to Schizosaccharomyces pombe $\mathrm{PHO} 2$ and Saccharomyces cerevisiae PHO13. Biochemistry 41: 7841-7848.

Opperdoes FR. 1987. Compartmentation of carbohydrate metabolism in trypanosomes. Annu Rev Microbiol 41: 127-151.

Opperdoes FR, Szikora JP. 2006. In silico prediction of the glycosomal enzymes of Leishmania major and trypanosomes. Mol Biochem Parasitol 147: 193-206.

Parsons M, Worthey EA, Ward PN, Mottram JC. 2005. Comparative analysis of the kinomes of three pathogenic Trypanosomatids: Leishmania major, Trypanosoma brucei and Trypanosoma cruzi. BMC Genomics 6: 127. doi: 10.1186/ 1471-2164-6-127.

Paterou A, Walrad P, Craddy P, Fenn K, Matthews K. 2006. Identification and stage-specific association with the translational apparatus of TbZFP3, a ccch protein that promotes trypanosome life cycle development. I Biol Chem 281: 39002-39013.

Pomerening JR, Sontag ED, Ferrell JE Jr. 2003. Building a cell cycle oscillator: Hysteresis and bistability in the activation of Cdc2. Nat Cell Biol 5: 346-351.

Pusnik M, Small I, Read LK, Fabbro T, Schneider A. 2007. Pentatricopeptide repeat proteins in Trypanosoma brucei function in mitochondrial ribosomes. Mol Cell Biol 27: 6876-6888.

Reddy VS, Singh AK, Rajasekharan R. 2008. The Saccharomyces cerevisiae PHM8 gene encodes a soluble magnesium-dependent lysophosphatidic acid phosphatase. I Biol Chem 283: 8846-8854.

Selengut JD, Levine RL. 2000. MDP-1: A novel eukaryotic magnesium-dependent phosphatase. Biochemistry 39: 83158324.

Siniossoglou S, Hurt EC, Pelham HR. 2000. Psrlp/Psr2p, two plasma membrane phosphatases with an essential DXDX $(\mathrm{T} / \mathrm{V})$ motif required for sodium stress response in yeast. $J$ Biol Chem 275: 19352-19360.

Sogin ML, Elwood HJ, Gunderson JH. 1986. Evolutionary diversity of eukaryotic small-subunit rRNA genes. Proc Natl Acad Sci 83: 1383-1387.

Stuart KD, Schnaufer A, Ernst NL, Panigrahi AK. 2005. Complex management: RNA editing in trypanosomes. Trends Biochem Sci 30: 97-105.

Szöör B, Wilson J, McElhinney H, Tabernero L, Matthews KR. 2006. Protein tyrosine phosphatase TbPTP1: A molecular switch controlling life cycle differentiation in trypanosomes. J Cell Biol 175: 293-303.

Tasker M, Wilson J, Sarkar M, Hendriks E, Matthews K. 2000. A novel selection regime for differentiation defects demonstrates an essential role for the stumpy form in the life cycle of the African trypanosome. Mol Biol Cell 11: 1905-1917.

Terlecky SR, Legakis JE, Hueni SE, Subramani S. 2001. Quantitative analysis of peroxisomal protein import in vitro. Exp Cell Res 263: 98-106.

Teusink B, Walsh MC, van Dam K, Westerhoff HV. 1998. The danger of metabolic pathways with turbo design. Trends Biochem Sci 23: 162-169.

Verplaetse E, Rigden DJ, Michels PA. 2009. Identification, characterization and essentiality of the unusual peroxin 13 from Trypanosoma brucei. Biochim Biophys Acta 1793: 516527.

Wirtz E, Leal S, Ochatt C, Cross GA. 1999. A tightly regulated inducible expression system for conditional gene knock-outs and dominant-negative genetics in Trypanosoma brucei. Mol Biochem Parasitol 99: 89-101.
Xue Y, Zhou F, Zhu M, Ahmed K, Chen G, Yao X. 2005. GPS: A comprehensive www server for phosphorylation sites prediction. Nucleic Acids Res 33: W184-W187. doi: 10.1093/ nar/gki393. 


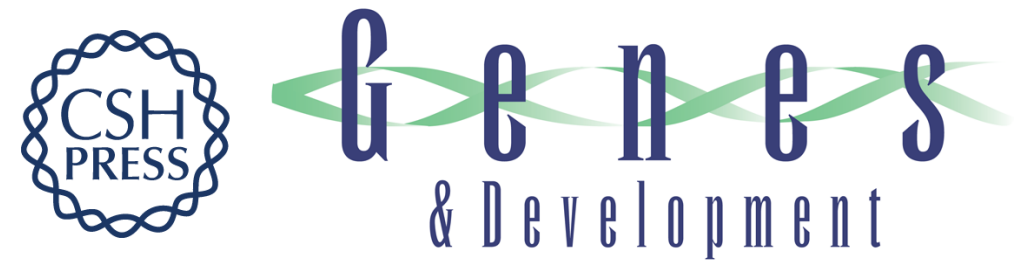

\section{A novel phosphatase cascade regulates differentiation in Trypanosoma brucei via a glycosomal signaling pathway}

Balázs Szöor, Irene Ruberto, Richard Burchmore, et al.

Genes Dev. 2010, 24:

Access the most recent version at doi:10.1101/gad.570310

\section{Supplemental http://genesdev.cshlp.org/content/suppl/2010/06/09/24.12.1306.DC1 \\ Material}

Related Content

Bistable Trypanosome Switch

Annalisa M. VanHook

Sci. Signal. June , 2010 3: ec186

References This article cites 52 articles, 19 of which can be accessed free at:

http://genesdev.cshlp.org/content/24/12/1306.full.html\#ref-list-1

Articles cited in:

http://genesdev.cshlp.org/content/24/12/1306.full.html\#related-urls

License Freely available online through the Genes \& Development Open Access option.

Email Alerting Receive free email alerts when new articles cite this article - sign up in the box at the top Service right corner of the article or click here.

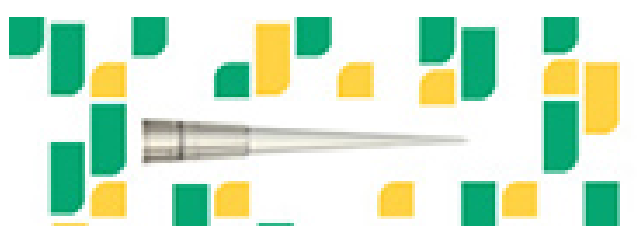

Focused on your science. 